\title{
Situación actual de la sarna e infecciones parasitarias en vicuñas (Vicugna vicugna) de la Región Cusco, Perú
}

\section{Current situation of mange and parasitic infections in vicuñas (Vicugna vicugna) of the Cuzco Region, Peru}

\author{
José M Angulo-Tisoc ${ }^{1,3.5}$, Joel I. Pacheco ${ }^{1,3}$, Víctor Vélez ${ }^{1}$, Wilber García ${ }^{1}$, \\ Henry Castelo ${ }^{2}$, Luis A. Gomez-Puerta ${ }^{3,4}$
}

\section{Resumen}

\begin{abstract}
El objetivo del presente estudio fue identificar y evaluar los casos de sarna causada por Sarcoptes scabiei y de endoparásitos en vicuñas silvestres y en cautiverio (Vicugna vicugna) de la Región Cusco, Perú. Se realizaron raspados de lesiones en piel y se tomaron muestras fecales durante la captura de vicuñas durante los chaccus. La frecuencia de $S$. scabiei fue de $1.9 \%$ (54/2826), siendo de 6.1\% (48/777) en silvestría y de $0.2 \%$ (6/ 2049 ) en cautiverio. Asimismo, se identificaron a F. hepatica (2.0\%), huevos tipo Strongylus (42.1\%), Nematodirus sp (6.8\%), N. spathiger (26.5\%), Trichuris sp (4.0\%), Eimeria spp (85.0\%), y Moniezia spp (2.7\%) en una muestra de 147 animales, siendo la mayor parte casos de monoparasitismo.
\end{abstract}

Palabra clave: sarna, Vicugna vicugna, camélidos, parásitos, Cusco

\footnotetext{
${ }^{1}$ Grupo de Investigación en Producción y Sanidad de Ganadería Altoandina, Estación IVITA Maranganí, Facultad de Medicina Veterinaria, Universidad Nacional Mayor de San Marcos, Cusco, Perú

${ }^{2}$ Proyecto de Aprovechamiento Sustentable de la Vicuña, Gobierno Regional Cusco, Perú

${ }^{3}$ Grupo de Investigación en Mejora de los Sistemas Productivos de Alpacas, Facultad de Medicina Veterinaria, Universidad Nacional Mayor de San Marcos, Lima, Perú

${ }^{4}$ Laboratorio de Epidemiología y Economía Veterinaria, Facultad de Medicina Veterinaria, Universidad Nacional Mayor de San Marcos, Lima, Perú

${ }^{5}$ E-mail: jangulot@unmsm.edu.pe; https://orcid.org/0000-0003-3238-5462
}

Recibido: 13 de octubre de 2020

Aceptado para publicación: 7 de abril de 2021

Publicado: 23 de junio de 2021

CLos autores. Este artículo es publicado por la Rev Inv Vet Perú de la Facultad de Medicina Veterinaria, Universidad Nacional Mayor de San Marcos. Este es un artículo de acceso abierto, distribuido bajo los términos de la licencia Creative Commons Atribución 4.0 Internacional (CC BY 4.0) [https:// creativecommons.org/licenses/by/4.0/deed.es] que permite el uso, distribución y reproducción en cualquier medio, siempre que la obra original sea debidamente citada de su fuente original 
The aim of this study was to identify and evaluate the cases of scabies caused by Sarcoptes scabiei and endoparasites in wild and captive vicuñas (Vicugna vicugna) from the Cusco Region, Peru. Scrapings of skin lesions were made and faecal samples were taken during the capture of vicunas during the chaccus. The frequency of S. scabiei was $1.9 \%(54 / 2826)$, being $6.1 \%(48 / 777)$ in the wild and $0.2 \%$ (6/2049) in captivity. Likewise, F. hepatica (2.0\%), Strongylus type eggs (42.1\%), Nematodirus sp (6.8\%), N. spathiger (26.5\%), Trichuris sp (4.0\%), Eimeria spp (85.0\%), and Moniezia spp (2.7\%) were found in a sample of 147 animals, being most cases monoparasitism.

Key words: scabies, Vicugna vicugna, camelids, parasites, Cusco

\section{INTRODUCCIÓN}

La vicuña (Vicugna vicugna) es un camélido sudamericano (CSA) silvestre que se distribuye en las ecorregiones de puna y cordillera desde el sur del Ecuador hasta el norte de Argentina, abarcando áreas de Perú, Bolivia y Chile (Acebes et al., 2018). La fibra que se obtiene de esta especie es muy valorada por su extrema suavidad, finura y costo en el mercado internacional (Wheeler y Hoces, 1997), por lo que ha sufrido gran presión de caza, llevándola casi al borde de la extinción; sin embargo, se pudo recuperar con una consecuente serie de esfuerzos y leyes tanto nacionales e internacionales orientadas a su conservación (Pérez, 1994; Quispe et al., 2009).

La mayor población mundial de vicuñas se encuentra en Perú, con una población total de 218000 cabezas en 2016. La Región Cusco ocupa el quinto lugar como poseedor de esta especie, siendo la Provincia de Canchis la que alberga el mayor porcentaje (DGFFS, 2014; Acebes et al., 2018).

Estudios sobre el endoparasitismo en vicuñas han sido reportados, dependiendo de factores tales como las condiciones geográficas (Gonzales-Mugaburu y Parra, 1956;
Bouts et al., 2003; Beltrán-Saavedra et al., 2011; Marcoppido et al., 2016), siendo a su vez relevante el compartir áreas de pastoreo o manejo con otras especies (Leguía, 1991; Cebra et al., 2014).

El ácaro Sarcoptes scabiei tiene una distribución mundial y es responsable de causar la sarna sarcóptica en diversas especies de mamíferos domésticos y silvestres, incluyendo al hombre (Pence y Ueckermann, 2002; Astorga et al., 2018). La sarna sarcóptica es considerada una afección cutánea con alta prevalencia y severidad en los CSA, que incluye a poblaciones de vicuñas (Garcilaso de la Vega, 1609; Fassi-Fehri, 1987; Lusat et al., 2009).

Dentro de los signos clínicos de esta parasitosis se encuentra el prurito con hipertermia, pápulas, pústulas con formación de costras ubicadas en diversas partes del cuerpo (Gomez-Puerta et al., 2013; Castillo, 2018). Además, producto de ello, se generan auto laceraciones, que deterioran la condición corporal y provocan emaciación progresiva, reduciendo la calidad y cantidad de fibra por el daño mecánico a la epidermis, e incluso provocando la muerte, con la consecuente reducción de poblaciones y pérdidas económicas para las comunidades locales (Arzamendia et al., 2012; Acebes et al., 
2018; Castillo, 2018). Bajo estas consideraciones, el objetivo del presente estudio fue evaluar la situación actual de la sarna y presencia de parásitos gastrointestinales en vicuñas de la Región Cusco.

\section{Materiales y Métodos}

El presente estudio fue realizado entre mayo y noviembre de 2018 y autorizado por el Servicio Nacional Forestal y de Fauna Silvestre del Perú (SERFOR) (N. ${ }^{\circ}$ RDG-2462018-MINAGRI-SERFOR-DGGSPFFS). Se recopilaron datos de 18 localidades de captura (Chaccu), de los cuales 11 chaccus fueron en silvestría y siete en cautiverio, con un total de 2826 animales capturados.

Todos los animales fueron evaluados por un médico veterinario para el diagnóstico de la presencia de sarna. La evaluación consistió en la revisión externa de los animales capturados, previo a la esquila o liberación, y la colecta de muestras de tejido epitelial de todo animal sospechoso a sarna. Asimismo, debido a las condiciones de manejo en el programa sanitario, solamente fue posible colectar muestras fecales de 147 animales para la evaluación coproparasitológica. Las muestras fecales fueron colectadas directamente del recto del animal, colocadas en bolsas de polietileno y almacenadas en recipientes térmicos con refrigerantes hasta su traslado al laboratorio para su procesamiento. Los animales fueron clasificados en tres edades según la evaluación dentaria: cría (dientes de leche), juvenil (dos dientes) y adulto (cuatro o más dientes), además de evidentes cambios en el tamaño corporal y la coloración del manto.

Para la identificación de sarna de animales clínicamente afectados, se realizaron raspados de piel en la zona circundante a la lesión y se conservaron en viales conteniendo etanol al $70 \%$. Luego, las muestras fueron sumergidas en una solución de hidróxido de potasio $(\mathrm{KOH})$ al $10 \%$ durante 12 horas.
El sedimento fue examinado por microscopía para detectar la presencia de ácaros. Los ácaros fueron identificados siguiendo las claves taxonómicas propuestas por Krantz y Walter (2009).

El examen coproparasitológico fue realizado utilizando las técnicas de flotación, sedimentación y McMaster, siguiendo los protocolos establecidos por Zajac y Conboy (2006). Todos los procedimientos se realizaron en el Laboratorio de Epidemiologia y Economía Veterinaria de la Facultad de Medicina Veterinaria de la Universidad Nacional Mayor de San Marcos, Lima.

Los resultados individuales de los animales se organizaron en una hoja de datos agrupándolos por tipo de manejo (silvestría y cautiverio), localidad (19 localidades), altitud (desde 3800 hasta $5200 \mathrm{msnm}$ ) y provincia (9 provincias del departamento del Cusco), registrando los casos positivos de sarna y endoparasitosis, además de consignar sus respectivas frecuencias individuales dentro de cada agrupación.

\section{Resultados}

De todas las vicuñas estudiadas $(\mathrm{n}=2826), 54(1.9 \%)$ de ellas presentaron lesiones compatibles con sarna sarcóptica, las cuales fueron confirmadas mediante el examen microscópico al detectar al S. scabiei en las muestras de raspado. La ocurrencia de sarna para vicuñas de silvestría fue 6.1\% (48/ 777) y para cautiverio $0.2 \%$ (6/2049) (Cuadro 1, Figura 1). Asimismo, dentro de los casos positivos a sarna, $85.1 \%(46 / 54)$ correspondió a animales adultos y $14.8 \%(8 / 54)$ a juveniles, en tanto que $40.7 \%$ (22/54) fueron machos y $59.2 \%(32 / 54)$ eran hembras.

La ocurrencia de sarna en vicuñas en silvestría se presentó en la provincia de Canchis, con excepción de Vela Punta y Los Andes que no evidenciaron casos. Por otro lado, en vicuñas en cautiverio solamente se 
Cuadro 1. Frecuencia de sarna en vicuñas de la Región Cusco, según el tipo de manejo y la provincia de origen

\begin{tabular}{|c|c|c|c|c|c|c|}
\hline \multirow{2}{*}{ Manejo } & \multirow{2}{*}{ Localidad } & \multirow{2}{*}{ Altitud } & \multirow{2}{*}{ Provincia } & \multirow{2}{*}{$\begin{array}{l}\text { Captura } \\
\text { (n) }\end{array}$} & \multicolumn{2}{|c|}{ Sarna } \\
\hline & & & & & $\mathrm{n}$ & $\%$ \\
\hline \multirow[t]{11}{*}{ Silvestría } & Sallani & $4800-5100$ & Canchis & 22 & 4 & 18.2 \\
\hline & Palccoyo & $4800-5100$ & Canchis & 47 & 5 & 10.6 \\
\hline & Ccuyo & $4600-5100$ & Canchis & 45 & 4 & 8.9 \\
\hline & Uchullucllo & $4700-5100$ & Canchis & 25 & 2 & 8.0 \\
\hline & Chilca & $5000-5200$ & Canchis & 100 & 8 & 8.0 \\
\hline & Sibina Sallma & $4800-5000$ & Canchis & 142 & 11 & 7.7 \\
\hline & Phacco & $4900-5200$ & Canchis & 164 & 11 & 6.7 \\
\hline & Llutuyo & $4800-5100$ & Canchis & 62 & 3 & 4.8 \\
\hline & Los Andes & $4500-4800$ & Canchis & 50 & 0 & 0 \\
\hline & Vela Punta & $4800-5100$ & Canchis & 50 & 0 & 0 \\
\hline & Quico & $4800-5200$ & Paucartambo & 70 & 0 & 0 \\
\hline Sub total & & & & 777 & 48 & 6.2 \\
\hline \multirow[t]{7}{*}{ Cautiverio } & Llusco & $3900-4100$ & Chumbivilcas & 312 & 6 & 1.9 \\
\hline & Tawapalcca & $3800-4500$ & Espinar & 100 & 0 & 0 \\
\hline & Quehue & $4000-4800$ & Canas & 637 & 0 & 0 \\
\hline & Pomacanchi & $3800-4600$ & Acomayo & 448 & 0 & 0 \\
\hline & $\begin{array}{l}\text { Yanque Lacca } \\
\text { lacca }\end{array}$ & $4200-4600$ & Paruro & 398 & 0 & 0 \\
\hline & Yanacancha & $4800-5100$ & Quispicanchi & 42 & 0 & 0 \\
\hline & Pumacancha & $4000-4600$ & Anta & 112 & 0 & 0 \\
\hline Sub total & & & & 2049 & 6 & 0.2 \\
\hline Total & & & & 2826 & 54 & 1.9 \\
\hline
\end{tabular}

presentó en la localidad de Llusco, provincia de Chumbivilcas (Cuadro 1, Figura 1).

De las 147 muestras fecales de vicuñas, $125(85.0 \%)$ fueron positivos a ooquistes de Eimeria spp, $62(42.1 \%)$ a huevos tipo Strongylus (HTS), $39 \quad(26.5 \%)$ a Nematodirus spathiger, $10(6.8 \%)$ a Nematodirus sp, $6(4.0 \%)$ a Trichuris sp, 4 (2.7\%) a Moniezia spp y $3(2.0 \%)$ a Fasciola hepatica.
Según la identificación de parásitos y edad del animal, la presencia de Eimeria spp fue alta en vicuñas adultas $(80 \%)$ y crías $(100 \%)$. Asimismo, la ocurrencia de HTS en juveniles y adultos fue superior al $40.0 \%$. De otra parte, no se presentó casos de $F$. hepatica y Trichuris sp en crías (Cuadro 2).

La parasitosis fue mayor en crías $(55 \%$; $11 / 20)$ que en juveniles y adultos $(31.9 \% ; 15 /$ 47 y $35.0 \%$; 28/80, respectivamente). Por 
Cuadro 2. Perfil de parásitos gastrointestinales (\%) en vicuñas manejadas bajo sistemas de silvestría y cautiverio en la Región Cusco, Perú, según la edad y sexo

\begin{tabular}{lcccccc}
\hline Parásito & $\begin{array}{c}\text { Cría } \\
(\mathrm{n}=20)\end{array}$ & $\begin{array}{c}\text { Juvenil } \\
(\mathrm{n}=47)\end{array}$ & $\begin{array}{c}\text { Adulto } \\
(\mathrm{n}=80)\end{array}$ & $\begin{array}{c}\text { Macho } \\
(\mathrm{n}=65)\end{array}$ & $\begin{array}{c}\text { Hembra } \\
(\mathrm{n}=82)\end{array}$ & $\begin{array}{c}\text { Total } \\
\text { positivo }\end{array}$ \\
\hline F. hepatica & 0 & 4.2 & 1.2 & 3.0 & 1.2 & 2.0 \\
HTS $^{1}$ & 10.0 & 42.5 & 50.0 & 46.1 & 39.0 & 42.1 \\
Nematodirus $\mathrm{sp}$ & 5 & 12.7 & 3.7 & 7.6 & 6.0 & 6.8 \\
N. spathiger & 25.0 & 38.2 & 20.0 & 30.7 & 23.1 & 26.5 \\
Trichuris $\mathrm{sp}$ & 0 & 4.2 & 5.0 & 3.0 & 4.8 & 4.0 \\
Eimeria $\mathrm{spp}$ & 100 & 87.2 & 80.0 & 89.2 & 81.7 & 85.0 \\
Moniezia $\mathrm{spp}$ & 5.0 & 4.2 & 1.2 & 3.0 & 2.4 & 2.7 \\
\hline
\end{tabular}

${ }^{1}$ Huevo tipo Strongylus

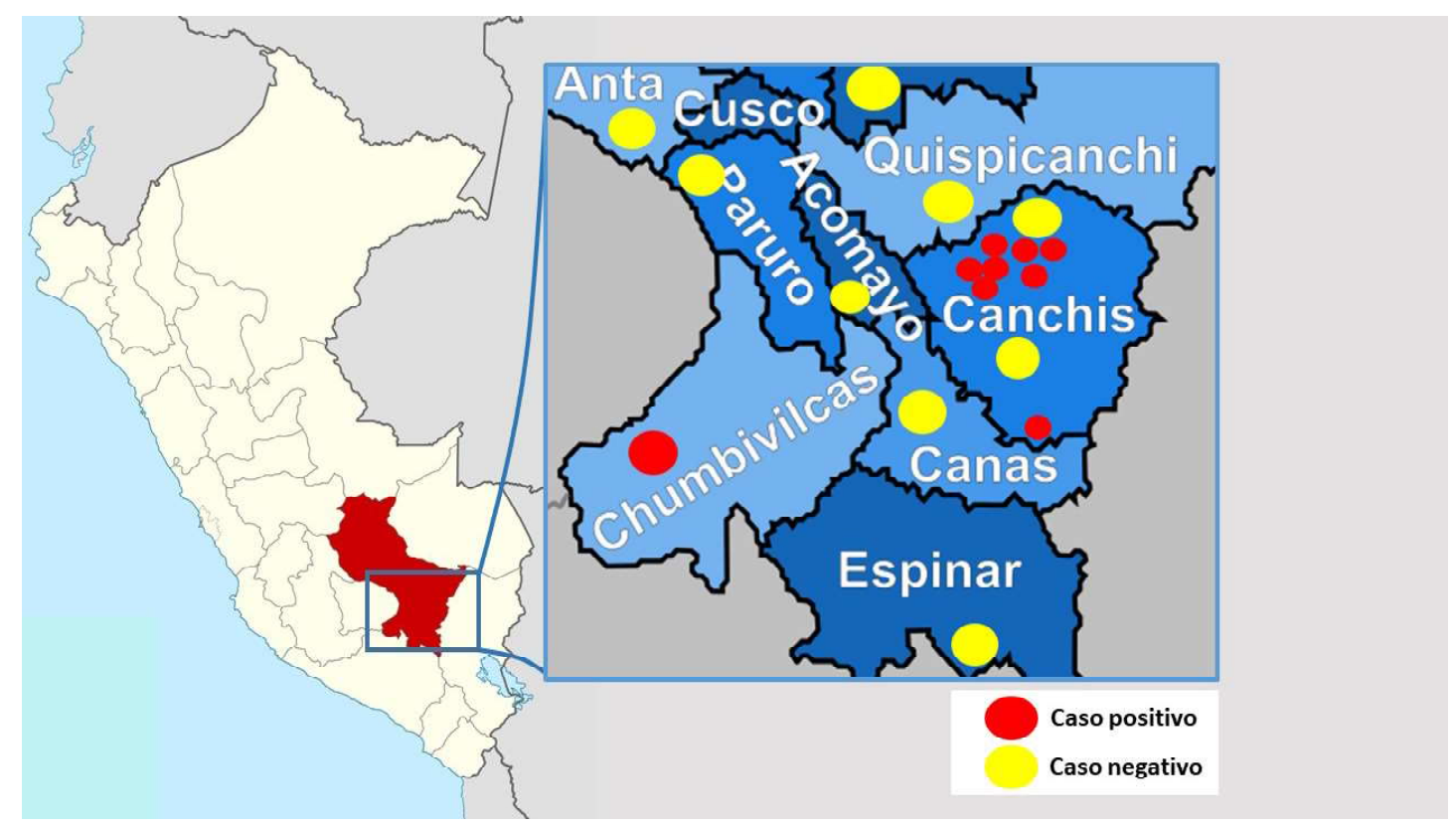

Figura 1. Distribución espacial de sarna sarcóptica en vicuñas de la Región Cusco (Ver Cuadro 1) 
Cuadro 3. Tipo de parasitismo en vicuñas manejadas bajo sistemas de silvestría y cautiverio en la Región Cusco, Perú, según la edad y sexo (n=147)

\begin{tabular}{|c|c|c|c|c|c|c|c|c|c|c|c|}
\hline & \multirow{2}{*}{ 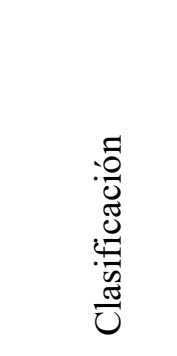 } & \multicolumn{2}{|c|}{ 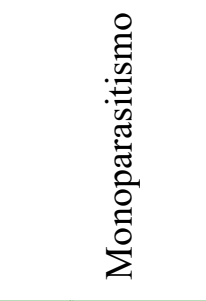 } & \multicolumn{2}{|c|}{ 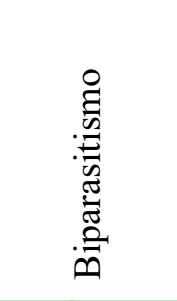 } & \multicolumn{2}{|c|}{ 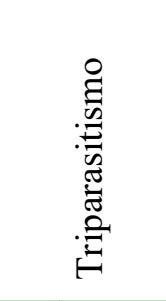 } & \multicolumn{2}{|c|}{ 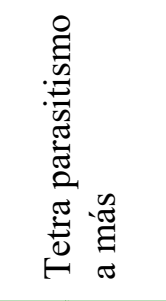 } & \multicolumn{2}{|c|}{ 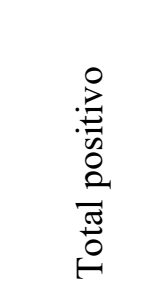 } \\
\hline & & $\mathrm{n}$ & $\%$ & $\mathrm{n}$ & $\%$ & $\mathrm{n}$ & $\%$ & $\mathrm{n}$ & $\%$ & $\mathrm{n}$ & $\%$ \\
\hline \multirow[t]{3}{*}{ Edad } & $\begin{array}{l}\text { Cría } \\
(n=20)\end{array}$ & 11 & 55.0 & 5 & 25.0 & 1 & 5.0 & 0 & 0 & 17 & 85.0 \\
\hline & $\begin{array}{l}\text { Juvenil } \\
(\mathrm{n}=47)\end{array}$ & 15 & 31.9 & 18 & 38.2 & 6 & 12.7 & 7 & 14.8 & 46 & 97.8 \\
\hline & $\begin{array}{l}\text { Adulto } \\
(\mathrm{n}=80)\end{array}$ & 28 & 35.0 & 28 & 35.0 & 11 & 13.7 & 4 & 5.0 & 71 & 88.7 \\
\hline \multirow[t]{2}{*}{ Sexo } & $\begin{array}{l}\text { Macho } \\
(\mathrm{n}=65)\end{array}$ & 23 & 35.3 & 24 & 36.9 & 11 & 16.9 & 3 & 4.6 & 61 & 93.8 \\
\hline & $\begin{array}{l}\text { Hembra } \\
(\mathrm{n}=82)\end{array}$ & 31 & 37.8 & 27 & 32.9 & 7 & 8.5 & 8 & 9.7 & 73 & 89.0 \\
\hline \multirow[t]{2}{*}{ Manejo } & $\begin{array}{l}\text { Cautiverio } \\
(\mathrm{n}=44)\end{array}$ & 23 & 52.2 & 6 & 13.6 & 3 & 6.8 & 1 & 2.2 & 33 & 75.0 \\
\hline & $\begin{array}{l}\text { Silvestría } \\
(\mathrm{n}=103)\end{array}$ & 31 & 30.0 & 45 & 43.6 & 15 & 14.5 & 10 & 9.7 & 101 & 98.0 \\
\hline
\end{tabular}

otro lado, el monoparasitismo fue mayor en hembras $(37.8 \% ; 31 / 82)$ y en animales en cautiverio (52.2\%; 23/44) (Cuadro 3). El biparasitismo en silvestría fue mayor que en cautiverio con $43.6 \%(45 / 103)$ y $13.6 \%(6 /$ 44), respectivamente. Para el caso de triparasitismo, la frecuencia fue mayor en adultos $(13.7 \%$; 11/80), animales machos $(16.9 \% ; 11 / 65)$ y silvestría $(14.5 \% ; 15 / 103)$. Finalmente, el tetraparasitismo presentó una mayor frecuencia en juveniles $(14.8 \% ; 7 / 47)$, hembras $(9.7 \% ; 8 / 82)$ y silvestría $(9.7 \% ; 10 /$ 103) (Cuadro 3).

\section{Discusión}

El presente estudio describe la situación actual de la sarna y endoparásitos en vicuñas de la Región Cusco. La frecuencia total de sarna en vicuñas fue de $1.9 \%$, en tanto que el $90.4 \%$ de los animales evaluados fueron positivos al menos a un endoparásito, siendo para ambas frecuencias la provincia de Canchis la que concentró el mayor porcentaje de casos (Cuadro 1), debido a que la mayor población de vicuñas y el mayor número de capturas se realiza en dicha Provincia. 
La frecuencia obtenida de sarna para el presente estudio fue inferior a las obtenidas en otros estudios realizados en el país. Por ejemplo, Unzueta (2018) analizó muestras de vicuñas de las provincias de Andahuaylas y Aymaraes, Región Apurímac y obtuvo una frecuencia de 9.4\% (69/733). Asimismo, dos estudios realizados en vicuñas de la Reserva Nacional 'Pampas Galeras' reportaron una frecuencia de sarna de $12.0 \%$ (24/ 200) (Gomez-Puerta et al., 2013) y $26.7 \%$ (1646/6162) (Bujaico y Zúñiga, 2015). La frecuencia de $1.9 \%(54 / 2826)$ del presente estudio podría deberse a factores como el manejo sanitario de los animales. Estudios realizados en Argentina reportan frecuencias de 0.8 y 9.0\% (Arzamendia et al., 2012; Aráoz et al., 2016), en tanto que en Bolivia se encontró frecuencias entre 5.6 y 46.2\% (BeltránSaavedra et al., 2011; Ruíz, 2016).

Se encontró una frecuencia de $6.1 \%$ de sarna en vicuñas manejadas en silvestría (Cuadro 1). Unzueta (2018) y Bujaico y Zúñiga (2015), reportaron 9.4 y $26.7 \%$, respectivamente para animales bajo este sistema de manejo, valores más elevados que en el presente estudio. Del mismo modo, en Argentina, Arzamendia et al. (2012) reportaron una prevalencia de $0.8 \%$ en poblaciones silvestres.

La variabilidad de resultados podría deberse a causas multifactoriales, como la carencia de condiciones adecuadas durante el proceso de manejo y captura, incluyendo el hacinamiento de grupos que en forma natural se encuentran distanciados (Gomez-Puerta et al., 2013; Castillo, 2018). Asimismo, el uso irresponsable de antiparasitarios se suma al riesgo de que la enfermedad no sea tratada correctamente y con riesgo de resistencia (Gomez-Puerta et al., 2013; Astorga et al., 2018; Castillo, 2018). Sin embargo, la transmisión podría depender de la condición social de la especie (Fthenakis et al., 2001; DevenishNelson et al., 2014; Ezenwa et al., 2016).
Por otro lado, la baja presentación de sarna en cautiverio del presente estudio podría deberse al manejo sanitario que se realiza en cada chaccu, ya sea sanitario o de esquila, puesto que anualmente se administran dosis de ivermectina a todos los animales capturados. De otra parte, para el caso de silvestría, Marcoppido et al. (2016) resaltan la selección natural de animales sanos y fuertes, además del fortalecimiento de la población para justificar la baja frecuencia; sin embargo, los impactos de esta enfermedad en poblaciones silvestres, sus mecanismos involucrados en la dinámica ecoepidemiológica y los riesgos para el ecosistema aún no están claros (Astorga et al., 2018).

La frecuencia de animales con sarna fue mayor en hembras (59.2\%) que en machos (40.7\%); resultado diferente al reportado por Unzueta (2018), quién registró una mayor frecuencia en machos. De otra parte, los resultados del presente estudio coinciden con los de este autor con relación a la edad, donde la mayor frecuencia de casos de sarna se presentó en animales adultos. No obstante, la variabilidad de la asociación al sexo o edad con los casos de sarna puede estar asociada al agente patógeno, al hospedero y al medio ambiente (Wobeser, 2009).

Los hallazgos de casos de sarna se encuentran bastante focalizados, principalmente en la provincia de Canchis, con frecuencias que oscilan entre 4.8 y $18.2 \%$, según la localidad. Esto podría deberse a que las áreas donde habitan las vicuñas son compartidas con camélidos domésticos, siendo S. scabei var. aucheniae el más común para camélidos de América del Sur. En el país se reportan casos de sarna hasta en el 40.0 y $25.0 \%$ en alpacas y llamas, respectivamente (Leguía, 1991; INEI, 2012; Sisson y Cebra, 2014). La transmisión entre estas especies puede ocurrir al compartir revolcaderos, estercoleros y lugares de pastoreo (Pedersen et al., 2007; Arzamendia et al., 2012). 
La alta endoparasitosis reportada concuerda con Quispe (2011) y Arias (2020), quienes obtuvieron en Tacna y Cusco una prevalencia de 80.8 y $84.4 \%$, respectivamente. Asimismo, en Bolivia, varios estudios reportan niveles que sobrepasan el $70.0 \%$, llegando incluso al 100\% (Beltrán-Saavedra et al., 2011; Martela, 2016; Ruiz, 2016); sin embargo, en Argentina, Marcoppido et al. (2016) detectaron porcentajes por debajo de $50.0 \%$ de carga parasitaria. Si bien la mayor frecuencia de endoparásitos se presentó en silvestría (Cuadro 2), Marcoppido et al. (2016) encontraron valores por debajo del $50.0 \%$ en este tipo de manejo.

Dentro de los parásitos identificados, Eimeria spp fue el más frecuente $(85.0 \%)$, siendo de $100 \%$ en las crías (Cuadro 2), lo cual coincide en edad con lo reportado por Arias (2020). Eimeria spp es considerado un patógeno importante involucrado en la muerte neonatal de alpacas cuando se presentan coinfecciones junto a Clostridium perfrigens (Palacios et al., 2006; Rosadio et al., 2010). No obstante a estos hallazgos, las tasas de vicuñas afectadas por este parásito son muy variables (Bouts et al., 2003; Quispe, 2011; Cafrune et al., 2014; Marcoppido et al., 2016). Asimismo, al igual que en los camélidos domésticos, se podría presentar casos de coccidiosis fatal (Dubey, 2018).

En tanto, según las especies identificadas, E. punoensis ha sido la coccidia más frecuente (Bouts et al., 2003; BeltránSaavedra et al., 2011; Cafrune et al., 2014; Martela, 2016; Ruiz, 2016; Arias, 2020). Para el caso de E. alpacae, los porcentajes reportados variaron entre 37.8 a 90.6\% (BeltránSaavedra et al., 2011; Cafrune et al., 2014; Martela, 2016; Ruiz, 2016), mientras que para E. lamae fueron entre 8.1 y $34.4 \%$ (BeltránSaavedra et al., 2011; Cafrune et al., 2014; Martela, 2016) y para E. macusaniensis 1legó hasta 39\% (Beltrán-Saavedra et al., 2011; Cafrune et al., 2014; Martela, 2016). Finalmente, E. peruviana ha sido reportada con $21.6 \%$ (Martela, 2016) y E. ivitaensis con $1.2 \%$ (Cafrune et al., 2014).
En el presente estudio se reportan dos casos de $F$. hepatica en animales juveniles y uno en un adulto (Cuadro 2). De otra parte, Samamé et al. (2016) reportaron una mayor frecuencia en juveniles frente a adultos, con valores de hasta 32.9\% (Gonzales-Mugaburu y Parra, 1956; Cafrune et al., 1996, 2004; Martela 2016; Samamé et al., 2016). Por otro lado, en alpacas está reportado la presencia de fasciolosis aguda y subaguda, pudiendo morir sin evidenciar signos clínicos (Leguía, 1997), lo que también podría suceder en vicuñas al margen del bajo porcentaje de hallazgo.

Respecto a los factores de presentación de fasciolosis, se encuentra la zona geográfica del presente estudio, con altitudes que llegan a superar los $4000 \mathrm{msnm}$, donde se evidencia la presencia de caracoles que albergan formas larvarias de dístoma hepático, posiblemente producto del calentamiento global (Londoñe et al., 2009; Samamé et al., 2016). Asimismo, la alta resistencia de $F$. hepatica a bajas temperaturas por periodos prolongados, sumado al hábitat compartido con especies domésticas como ovinos, bovinos y CSA domésticos, facilitarían la presentación de fasciolosis en las vicuñas (Leguía, 1991, 1997; Cordero del Campillo et al., 1999), existiendo limitantes para su tratamiento por las condiciones de crianza.

\section{Conclusiones}

- La presencia de sarna fue mayor en silvestría con $6.1 \%$ en comparación con la crianza en cautiverio de $0.2 \%$.

- Los endoparásitos identificados fueron Fasciola hepatica, huevos tipo Strongylus, Nematodirus sp, $N$. spathiger, Trichuris sp, Eimeria spp y Moniezia spp.

- El porcentaje de monoparasitismo es alto y al parecer no influenciado por el sistema de manejo ni el sexo. 


\section{Literatura Citada}

1. Acebes P, Wheeler J, Baldo J, Tuppia P, Lichtenstein G, Hoces D, Franklin WL. 2018. Vicugna vicugna (errata version published in 2019). The IUCN Red List of Threatened Species 2018: e.T22956A145360542. doi: 10.2305/ IUCN.UK.2018-2.RLTS.T22956A145360542.en

2. Aráoz V, Aguirre DH, Viñabal AE, Acuña, Abalos M, Micheloud J. 2016. Descripción clínico-patológica en brotes de sarna sarcóptica en vicuñas (Vicugna vicugna) y llamas (Lama glama) de la provincia de Jujuy, INTA Argentina. En: $X$ Reunión Argentina de Patología Veterinaria. Santa Fé.

3. Arias C. 2020. Parasitas e seu impacto no chaccu de vicunhas em comunidades andinas no Peru: uma abordagem One Health. Tesis de Maestría. São Paulo: Univ. Estadual Paulista. 27 p.

4. Arzamendia $Y$, Neder LE, Marcoppido G, Ortiz F, Arce M, Lamas HE, Vilá BL. 2012. Effect of the prevalence of ectoparasites in the behavioral patterns of wild vicuñas (vicugna vicugna). J Camelid Sci 5: 105-117.

5. Astorga F, Carver S, Almberg E, Sousa GR, Wingfield K, Niedringhaus KD, Wick PV, et al. 2018. International meeting on sarcoptic mange in wildlife, June 2018, Blacksburg, Virginia, USA. Parasite Vector 11: 449. doi: 10.1186/ s13071-018-3015-1

6. Beltrán-Saavedra LF, NallarGutierrez, R, Ayala G, Limachi JM, Gonzales-Rojas JL. 2011. Estudio sanitario de vicuñas en silvestría del área natural de manejo integrado nacional Apolobamba, Bolivia. Ecología en Bolivia 46: 14-27.

7. Bouts T, Fox MT, Scheres G, Chavez A. 2003. Identification of gastrointestinal nematodes and coccidia in wild vicunas Lama vicugna in Pampa Galeras, Peru. Internationalen Symposiums uber die
Erkrankungen der Zoo und Wildtiere. Rome, Italy.

8. Bujaico N, Zúñiga M. 2015. Control y tratamiento de sarna (escabiosis) en vicuñas de la comunidad campesina de Lucanas, Reserva Nacional Pampa Galeras. Ayacucho Perú. Cienc Desarro 18: 31-36. doi: 10.21503/Cienciay Desarro1lo.-2015.v18i2.04

9. Cafrune MM, Rebuffi GE, Gaido AB, Aguirre DH. 1996. Fasciola hepatica in semi-captive vicuñas (Vicugna vicugna) in northwest Argentina. Vet Rec 139: 97. doi: 10.1136/vr.139.4.97-a

10. Cafrune MM, Aguirre DH, Freytes I. 2004. Fasciolosis en vicuñas (Vicugna vicugna) en semi-cautiverio de Molinos, Salta, Argentina, con notas de otros helmintos en este hospedador. Vet Arg 21:513-520.

11. Cafrune M, Romero S, Aguirre D. 2014. Prevalence and abundance of Eimeria spp. infection in captive vicuñas (Vicugna vicugna) from the Argentinean Andean Altiplano. Small Ruminant Res 120: 150-154. doi: 10.1016/j.smallrumres.2014.04.013

12. Castillo H. 2018. Implicancias sanitarias en el manejo sostenible de camélidos sudamericanos silvestres. Sarna y otras enfermedades producidas por parásitos. GECS News 7: 11-24.

13. Cebra CK, Anderson D, Tibary A, Van Saun R, Johnson LW. 2014. Disorders of the digestive system. In: Llama and alpaca care, medicine, surgery, reproduction, nutrition, and health care. Saint Louis: Saunders Elsevier. p 477-536.

14. Cordero del Campillo M, Rojo-Vásquez FD, Martínez AR, Sánchez MC, Hernández, S, Navarrete I, Diez, P, et al. 1999. Parasitología veterinaria. España: McGraw Hill Interamericana. 968 p.

15. Devenish-Nelson ES, Richards SA, Harris S, Soulsbury C, Stephens PA, 2014. Demonstrating frequencydependent transmission of sarcoptic mange in red foxes. Biol Letters 10: 20140524. doi: 10.1098/rsbl.2014.0524 
16. DGFFS. 2014. Censo poblacional de vicuñas 2012. Ministerio de Agricultura y Riego. Lima Perú.

17. Dubey JP. 2018. A review of coccidiosis in South American camelids. Parasitol Res 117: 1999-2013. doi: 10.1007/s00436018-5890-y

18. Ezenwa VO, Ghai RR, Mckay AF, Williams $A E$. 2016. Group living and pathogen infection revisited. Curr Opin Behav Sci 12: 66-72. doi: 10.1016/ j.cobeha.2016.09.006

19. Fassi-Fehri MM. 1987. Las enfermedades de los camélidos. Rev Sci Tech OIE 6: 355-373.

20. Fthenakis GC, Karagiannidis A, Alexopoulos $C$, Brozos $C$, Papadopoulos E. 2001. Effects of sarcoptic mange on the reproductive performance of ewes and transmission of Sarcoptes scabiei to newborn lambs. Vet Parasitol 95: 63-71. doi: 10.1016/ s0304-4017(00)00417-9

21. Garcilaso De la Vega I. 1609. Comentarios reales. Libro octavo. Lisboa, Portugal.

22. Gomez-Puerta LA, Olazabal J, Taylor CE, Cribillero NG, López-Urbina MT, Gonzales AE. 2013. Sarcoptic mange in vicuña (Vicugna vicugna) population in Perú. Vet Rec 173: 269. doi: 10.1136/ vr.101320

23. Gonzales-Mugaburu L, Parra B. 1956. Nota sobre el hallazgo de algunos helmintos en vicuña (Vicugna vicugna). Rev Peru Med Exp Salud Publica 10: 153-154.

24. [INEI] Instituto Nacional de Estadistica e Informática. 2012. IV Censo Nacional Agropecuario 2012. [Internet]. Disponible en: http://censos.inei.gob.pe/ Cenagro/redatam/\#

25. Krantz GW, Walter DE. 2009. A manual of acarology. $3^{\text {rd }}$ ed. USA: Texas Tech University Press. 807 p.

26. Leguía G 1991. The epidemiology and economic impact of llama parasites. Parasitol Today 7: 54-56. doi: 10.1016/ 0169-4758(91)90190-y
27. Leguía G. 1997. Acute and subacute fasciolosis of alpacas (Lama pacos) and treatment with triclabendazole. Trop Anim Health Pro 29: 31-32. doi: 10.1007/ BF02632344

28. Londoñe P, Chávez A, Li O, Suárez F, Pezo D. 2009. Presencia de caracoles Lymnaeidae con formas larvarias de Fasciola hepatica en altitudes sobre los 4000 msnm en la sierra sur del Perú. Rev Inv Vet Perú 20: 58-65. doi: 10.15381/ rivep.v20i1.533

29. Lusat J, Morgan ER, Wall R. 2009. Mange in alpacas, llamas and goats in the UK: Incidence and risk. Vet Parasitol 163: 179-184. doi: 10.1016/j.vetpar.2009.04.007

30. Marcoppido G, Schapiro J, Morici G, Arzamendia Y, Vilä B. 2016. Coproparasitological evaluations of nematodes and coccidia in a wild vicuña (Vicugna vicugna) population in the argentinean andean altiplano. J Camelid Sci 9: 23-34.

31. Martela W. 2016. Identificación de parásitos gastrointestinales en poblaciones de vicuña (Vicugna vicugna) en tres regiones de Bolivia. Tesis de Ingeniero en Producción y Comercialización Agropecuaria. Bolivia: Univ. Mayor de San Andrés. $88 \mathrm{p}$.

32. Palacios CA, Perales RA, Chavera AE, Lopez MT, Braga WU, Moro M. 2006. Eimeria macusaniensis and Eimeria ivitaensis co-infection in fatal cases of diarrhoea in young alpacas (Lama pacos) in Peru. Vet Rec 158: 344345. doi: 10.1136/vr.158.10.344

33. Pedersen AB, Jones KE, Nunn $C L$, Altizer S. 2007. Infectious diseases and extinction risk in wild mammals. Conserv Biol Ser 21: 1269-1279. doi: 10.1111/ j.1523-1739.2007.00776.x

34. Pence DB, Ueckermann E. 2002. Sarcoptic mange in wildlife. Rev Sci Tech OIE 21:385-398.

35. Pérez W. 1994. La saga de la vicuña. Lima, Perú: CONCYTEC. 408 p. 
36. Quispe EC, Rodríguez TC, Iñiguez LR, Mueller JP. 2009. Producción de fibra de alpaca, llama, vicuña y guanaco en Sudamérica. Anim Genet Resour 45: 1-14. doi: 10.1017/S1014233909990277

37. Quispe HH. 2011. Estudio de parásitos externos y gastrointestinales en vicuñas (Vicugna vicugna mensalis) en el anexo Mamuta de la Provincia de Tarata en la Región Tacna. Tesis de Médico Veterinario y Zootecnista. Tacna: Univ. Nacional Jorge Basadre Grohmann. $85 \mathrm{p}$.

38. Rosadio R, Londoñe P, Pérez D, Castillo H, Véliz A, Llanco L, Yaya K, Maturrano L 2010. Eimeria macusaniensis associated lesions in neonate alpacas dying from enterotoxemia. Vet Parasitol 168: 116-120. doi: 10.1016/ j.vetpar.2009.10.010

39. Ruiz C. 2016. Identificación y caracterización de la presencia de ectoparásitos y endoparásitos en vicuñas (Vicugna vicugna) en comunidades de los departamentos de La Paz y Oruro. Tesis de Maestría. Bolivia: Univ. Mayor de San Andrés. 80 p.
40. Samamé LM, Chávez A, Pinedo R. 2016. Fascioliosis en vicuñas (Vicugna vicugna) de la sierra central del Perú. Rev Inv Vet Perú 27: 137-144. doi: 10.15381/rivep.v27i1.11459

41. Sisson M, Cebra C, 2014. Disorders of the skin. In: Llama and alpaca care, medicine, surgery, reproduction, nutrition, and health care. Saint Louis, USA: Saunders Elsevier. p 379-392.

42. Unzueta L. 2018. Sarna en vicuñas (Vicugna vicugna) en las provincias de Aymaraes y Andahuaylas de la Región Apurímac. Tesis de Médico Veterinario y Zootecnista. Apurímac, Perú: Univ. Nacional Micaela Bastidas de Apurímac. $124 \mathrm{p}$.

43. Wheeler J, Hoces D. 1997. Community participation, sustainable use, and vicuña conservation in Perú. Mt Res Dev 17: 283-287. doi: 10.2307/3673855

44. Wobeser G 2009. Fundamentos de las enfermedades de los animales silvestres. Ed Acribia. 284 p.

45. Zajac AM, Conboy GA. 2006. Veterinary clinical parasitology. $7 \mathrm{td}$ ed. Iowa, USA: Blackwell Publishing. 320 p. 